$\underline{\text { Case Reports }}$

\title{
Madurella Mycetomatis Infection Following Allogenic Stem Cell Transplantation for Aplastic Anemia
}

\author{
Sanjeev Kumar Sharma ${ }^{1}$, Anjan Mukherjee ${ }^{2}$, Avinash Kumar Singh ${ }^{1}$, Tulika Seth ${ }^{1}$, Suman Kumar ${ }^{1}$,
} Pravas Mishra ${ }^{1}$, Immaculata Xess ${ }^{2}$, Somesh Gupta ${ }^{3}$, Manoranjan Mahapatra ${ }^{1}$ and Haraprasad Pati ${ }^{1}$

Departments of Hematology ${ }^{1}$, Microbiology $^{2}$, and Dermatology ${ }^{3}$

All India Institute of Medical Sciences, New Delhi, India

Correspondence to: Tulika Seth, MD. Department of Hematology, All India Institute of Medical Sciences, New Delhi, India. Tel: 09868397236. E-mail: tuliseth@yahoo.com.

Competing interests: The authors have declared that no competing interests exist.

Published: June 14, 2012

Received: March 27, 2012

Accepted: May 25, 2012

Citation: Mediterr J Hematol Infect Dis 2012, 4(1): e2012038, DOI: 10.4084/MJHID.2012.038

This article is available from: http://www.mjhid.org/article/view/10298

This is an Open Access article distributed under the terms of the Creative Commons Attribution License (http://creativecommons.org/licenses/by/2.0), which permits unrestricted use, distribution, and reproduction in any medium, provided the original work is properly cited.

\section{Dear Editor,}

Eumycetoma is a slow growing fungal infection, which initially involves the skin and subcutaneous tissues and progresses to involve deeper structures. It is characterized clinically by multiple sinuses draining purulent exudate containing fungal granules. Since the foot is the most common site of initial localization of the lesion, the term "Madura foot" has been used to describe it. Strong clinical suspicion followed by categorization of lesion into eumycotic or actinomycotic by suitable culture is essential for diagnosis and effective therapy as delay in diagnosis may be limb or life threatening. We report here, a case of madura foot caused by Madurella mycetomatis, in a patient with aplastic anemia following allogenic peripheral blood stem cell transplantation with favourable clinical outcome.

A 22-years-old male was diagnosed as a case of very severe aplastic anaemia seven months back when he presented with fever and gum bleeding. He had HLA matched sibling and underwent allogenic peripheral blood stem cell transplantation. Conditioning regimen included fludarabine $30 \mathrm{mg} / \mathrm{m}^{2}$ for 6 days and cyclophosphamide $60 \mathrm{mg} / \mathrm{m}^{2}$ for 2 days. Graft versus host disease (GVHD) prophylaxis included anti-thymocyte globulin $30 \mathrm{mg} / \mathrm{kg} /$ day for 4 days, methotrexate $\left(10 \mathrm{mg} / \mathrm{m}^{2}\right)$ on day $+1,+3$ and +6 , and cyclosporine $100 \mathrm{mg}$ twice daily intravenously followed by oral cyclosporine with dose adjusted according to plasma cyclosporine levels (between 150$300 \mathrm{ng} / \mathrm{ml}$ ). He engrafted on day +9 (absolute neutrophil count $>0.5 \times 10^{9} / 1$ and unsupported platelet count $\left.>20 \times 10^{9} / 1\right)$. Two months post transplant patient noticed a small painless nodular swelling over the plantar surface of right foot. He was on cyclosporine at that time. There was no history of trauma or thorn prick injury. The swelling progressively increased in size in next two months with appearance of two discharging sinuses (Figure 1). Radiograph of right foot revealed soft tissue radio-opacity without involvement of underlying bony structures. There was discharge of black grains which on $\mathrm{KOH}$ mount examination under microscope (40X magnification) revealed presence of septate hyphae. The culture of the discharged granules in Sabouraud's dextrose agar (SDA) grew Madurella mycetomatis (Figure 2) after an incubation for 12 days. The patient was started on voriconazole (400 mg orally twice daily on first day followed by $200 \mathrm{mg}$ twice daily). The lesion was completely excised one week later. Biopsy smears 


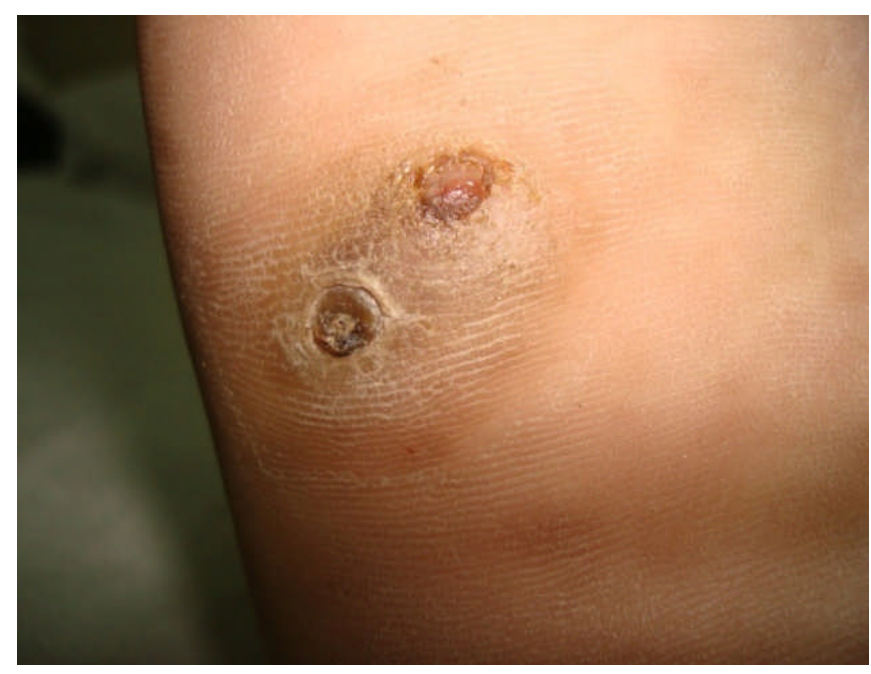

Figure 1. Lesion on the foot showing two discharging sinuses.

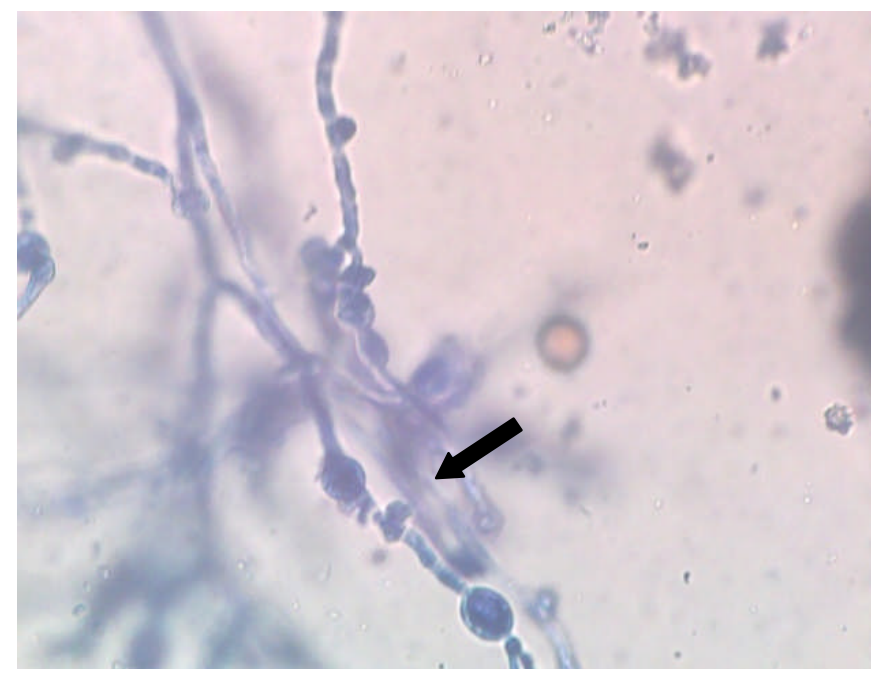

Figure 2. Wet mount from culture stained with Lactophenol Cotton Blue (LCB) showing brown pigmented septate hyphae with intercalary chlamydospores (arrow) characteristic of Madurella mycetomatis.

showed distinct brown-black colonies of the fungus having branching and septate hyphae embedded in matrix like material against a mixed inflammatory background. Voriconazole was continued and there is no recurrence of lesion three months post excision.

With the increasing number of patients undergoing transplantation, newer fungal infections are emerging and posing a diagnostic and therapeutic challenge. Mycetomatis is a chronic infection which initially involves cutaneous areas and progresses to involve deeper structures. Mycetoma presents as a slow growing indolent infection characterised by tumaefaction and multiple discharging sinuses. The etiological agents can be a variety of fungal agents (eumycetoma) or bacteria belonging to the actinomycetes group (actinomycetoma). Madurella mycetomatis is the worldwide predominant eumycetoma, followed by Scedosporium apiosperma, Scedosporium prolificans, and Madurella grisea. ${ }^{1}$
These fungi account for approximately 95\% of eumycetoma cases. Madurella mycetomatis (black grain mycetoma) is the most common eumycetoma in India. ${ }^{2,3}$ It is also endemic in Sudan, Central and South America and Indonesia. ${ }^{4}$ In contrast, Scedosporium (white grain mycetoma) is the commonest eumycetoma in North America. ${ }^{5}$ It is the most commonly reported eumycetoma in transplant recipients, which is associated with a high rate of dissemination and a high mortality. ${ }^{5}$ There is no data of madurella infection in transplant recipients except for two reported cases who received immunosuppressive therapy for renal transplantation (infected by Madurella grisea and Madurella mycetomatis, respectively). ${ }^{6,7}$ With the increasing number of patients undergoing transplants in tropical and subtropical countries, Madurella mycetomatis will be an emerging fungal infection in such a group of population.

The differential diagnosis of eumycetoma lesions include actinomycetoma, botryomycosis, cutaneous tuberculosis, fungal diseases such as blastomycosis and coccidioidomycosis, and cutaneous nocardiosis. An early diagnosis is essential since the disease has a progressive course with extension and damage to deeper tissues and internal organs, particularly more so in immunocompromised patients. Discharging sinus tracts are very characteristic of mycetoma and are helpful in making the clinical diagnosis. The cytological diagnosis of eumycetoma can be as accurate as histological diagnosis, and techniques such FNAC as well as imprint smears are simple, inexpensive and fairly reliable techniques without any obvious disadvantages. ${ }^{8}$

Early surgery can be curative for a small, localized eumycetoma lesion amenable to total excision and has been considered as a standard of care for such patients. ${ }^{9}$ Medical therapy of nonresectable or disseminated disease in immunocompromised patients is ineffective ${ }^{9}$ and may require aggressive debridement. Ketoconazole has been found effective for mycetoma caused by $M$. mycetomatis in at least $70 \%$ cases but is not an effective treatment for eumycetoma caused by Scedosporium species. The new second generation triazoles, including voriconazole and posaconazole have been tried as potential agents for eumycetoma, with some success. ${ }^{10,11}$

Our patient developed nodular lesion on the plantar surface of right foot two months post transplant which slowly progressed and resulted in discharging sinuses. Biopsy revealed infection by $M$. mycetomatis. This is probably the first reported case of $M$. mycetomatis infection in a patient following allogenic hematopoietic stem cell transplantation. Early complete excision of the lesion resulted in complete cure. With increasing number of patients undergoing stem cell 
transplantation in tropical countries, eumycetoma caused by $M$. mycetomatis is one of the emerging fungal infections among transplant recipients, because of prolonged duration of immunosuppression. Since the fungus has poor response to antifungal drugs, early surgical debridement is very essential before the

\section{References:}

1. McGinnis MR, Fader RC: Mycetoma: a contemporary concept. Infect Dis Clin North Am 1988; 2:939-954. PMid: 3062096

2. Chakraborti A, Singh K: Mycetoma in Chandigarh and surrounding areas. Indian J Med Microbiol 1998; 16:64-65.

3. Padhi S, Uppin SG, Uppin MS, et al: Mycetoma in South India: retrospective analysis of 13 cases and description of two cases caused by unusual pathogens: Neoscytalidium dimidiatum and Aspergillus flavus. Int J Dermatol 2010; 49:1289-1296. doi: http://dx.doi.org/10.1111/j.1365-4632.2010.04610 PMid: 20964650

4. Ahmed A, Adelmann D, Fahal A, et al: Environmental occurrence of Madurella mycetomatis, the major agent of human eumycetoma in Sudan. J Clin Microbiol 2002; 40:1031-1036. doi: http://dx.doi.org/10.1128/JCM.40.3.1031-1036.2002 PMid: 11880433

5. Husain $S$, Muñoz $P$, Forrest $G$, et al: Infections due to Scedosporium apiospermum and Scedosporium prolificans in transplant recipients: Clinical characteristics and impact of antifungal agent therapy on outcome. Clin Infect Dis 2005; 40:8999. doi: http://dx.doi.org/10.1086/426445 PMid: 15614697

6. O'Riordan E, Denton J, Taylor PM, et al: Madura foot in the UK: fungal osteomyelitis after renal transplantation. Transplantation 2002; 73:151-153. PMid: 11792997

7. Meis J, Schouten RA, Verweij PE, et al: Atypical presentation of infection spreads to involve deeper structures.

Acknowledgements. We are thankful to Dr Sandeep Sharma and Aashna Sharma for formatting the manuscript.
Madurella mycetomatis mycetoma in a renal transplant patient. Transpl Infect Dis 2000; 2:96-98. doi: http://dx.doi.org/10.1034/j.1399-3062.2000.020208.x PMid:11429018

8. Afroz N, Khan N, Siddiqui FA, Rizvi M: Eumycetoma versus actinomycetoma: Diagnosis on cytology. J Cytol 2010; 27:133135. doi: http://dx.doi.org/10.4103/0970-9371.73297 PMid: 21157564

9. Welsh O, Salinas MC, Rodriguez MA, et al: Treatment of eumycetoma and actinomycetoma. Curr Top Med Mycol 1995; 6:47-71. PMid: 8724241

10. McGinnis MR, Pasarell L: In vitro testing of susceptibilities of filamentous ascomycetes to voriconazole, itraconazole, and amphotericin B, with consideration of phylogenetic implications. J Clin Microbiol 1998; 36:2353-2355. PMid: 9666022

11. Van De Sande WW, Luijendijk A, Ahmed AO, et al: Testing of the in vitro susceptibilities of Madurella mycetomatis to six antifungal agents by using the Sensititre system in comparison with a viability-based 2, 3-bis (2-methoxy-4-nitro-5-sulfophenyl)-5[(phenylamino) carbonyl]-2H-tetrazolium hydroxide (XTT) assay and a modified NCCLS method. Antimicrob Agents and Chemother 2005; 49:1364-1368 . doi: http://dx.doi.org/10.1128/AAC.49.4.1364-1368.2005 PMid: 15793113 